Environment Conservation Journal 14(1\&2) 1-7, 2013

ISSN 0972-3099 (Print) 2278-5124 (Online)

Abstracted and Indexed

\title{
Studies on solid waste generation and composition in the Residential area of Akhnoor town, District Jammu
}

\author{
Shalini Sharma $\bowtie$ and Subash C. Gupta
}

Received: 15.09 .2012

Accepted: 8.01.2013

\begin{abstract}
The present paper deals with the analysis of solid waste generation and composition within the municipal limits of Akhnoor town which starts from the main bridge on the river Chenab and extends up to Sohal-Sungal turn. For purpose of studies, the residential area was divided into four zones and from each zone, five houses were selected at random for the sampling and analysis of solid waste for a period of one year. Characterization and management of solid waste alongwith methods of disposal of Municipal Solid Waste (MSW) were studied to analyze its impact on the environment and people inhabiting the area. Proper disposal methods have also been suggested so that the environment in general and the population inhabiting the area in particular is saved from the hazardous effects of fast increasing menace of the waste.
\end{abstract}

Keywords: Solid Waste, MSW, Disposal, Hazardous

\section{Introduction}

At present, there are so many environmental issues which have grown in size and complexity day by day, thereby, threatening the survival of mankind on this planet earth and the problem of solid waste pollution is one of them. According to Santra (2001), solid waste is a conglomeration of dust, ash, vegetable, putrescible matter, paper, packing of all kinds, rags and other fabrics, glass and other combustible and non-combustible debris. In Jammu and Kashmir, like other Indian states, at most of the places, refuse is dispersed all along the roads, streets, drains, open spaces etc. As per records of the Jammu Municipal Corporation based on a survey conducted by Environmental Engineers of Government of India (GOI) Public Sector Undertaking RITES, 8 lakh population falling under its jurisdiction, has been generating 450 tonnes of solid waste/day. Municipal solid waste of Jammu contains $54 \%$ biodegradable, 14\% recyclable and remaining 32\% material on dry weight basis with 45\% moisture content (Daily Excelsior, June 26, 2006).Though a lot of work has been done on generation, composition and management of solid waste from India and abroad

\section{Author's Address}

Deptt of Environmental Sciences, Univ of Jammu, Jammu E-mail: sharmashalini1977@gmail.com by various workers, but no work seems to have been done on solid waste generated in the residential area of Akhnoor town.However, some workers like Rampal et al. (2002), Kour (2004), Rampal et al. (2005), Gupta et al. (2007), Gupta et al. (2008), Jaswal (2008) and Kewal (2010) have provided some fragmentary information on the generation and characteristics of waste of Jammu Municipality. The present study will help to generate information on the generation, composition and management of municipal solid waste and place before the management, the problems arising out of its improper disposal.

\section{Material and Methods}

\section{Study Area}

The present study was conducted within the municipal limits of Akhnoor town which starts from the main bridge on River Chenab and extends up to Sohal-Sungal turn. Geographically, Akhnoor lies at a latitude of $32.9^{0} \mathrm{~N}$ and longitude of $74.75^{\circ}$ E, situated in the North-West part of India and eastern part of Pakistan and is about 32Km from Jammu. It has a total area of $1.5 \mathrm{sq} \mathrm{Km}$ with a population of 11346. 


\section{Methodology}

For the purpose of waste collection, the study area was divided into four different zones. Thus selecting five houses from each zone twenty houses were selected from the study area for purpose of studies. The sampling was done over a period of one year, i.e., from June, 2007 to May, 2008. Monthly sampling of solid waste was done by collecting waste from each household, segregated into different components and weighed separately with the help of spring balance. The various components of waste collected for analysis were identified as paperware, cardboard, clothware, jute, foliage, cotton, wood, food and garbage (biodegradable); plasticware, metallicware, glassware, thermocoal, rubber, leather, egg shells and bones (non-biodegradable) and inert wastes.During each sampling, the weight of solid waste generated per day in each house and the number of members of the house was recorded. The average weight of solid waste per capita per day was calculated by using the formula-

Solid waste generation (Kg/capita/day) =

Solid waste generation per day at a particular household during 24 hours Total no. of residents of household during sampling

\section{Results and Discussion}

The results of 12 months data on solid waste generation and composition are presented in Table1, 2, 3 and 4. Comparative study of average solid waste (Kg/capita/month) generation at four different study zones, i.e. Zone-I to Zone -IV containing 5 houses each (viz. H.1 to H.5) during one year has been made (Table 5).A critical evaluation of Table- 5 has revealed that in the study area (i.e, Zone I to Zone-IV) the total solid waste generated (Kg/capita) was estimated to be 417.396 $\mathrm{Kg}$ with an average of $34.783 \pm 14.121 \mathrm{Kg}$ out of which $342.276 \mathrm{Kg}$ was contributed by biodegradable waste (82\%) comprising of paperware, card board, clothware, jute, foliage, cotton, wood, food/garbage, etc. Nonbiodegradable waste was calculated to be 64.416 $\mathrm{Kg} \mathrm{(15.4 \% )} \mathrm{comprising} \mathrm{of} \mathrm{plasticware,}$ metallicware, glassware, thermocoal, rubber, etc and $10.704 \mathrm{Kg}$ of inert waste (2.6\%) which comprised of hair, dust, pebbles, sand, gravels, etc with average values of $28.523 \pm 10.349$ $\mathrm{Kg}, 5.368 \pm 3.469 \mathrm{Kg}$ and $0.892 \pm 0.302 \mathrm{Kg}$, respectively. The per capita generation of these various components have been depicted in Table 15.An overall study has revealed maximum percentage of biodegradable solid waste (82\%) followed by non-biodegradable solid waste (15.4\%) and inert solid waste (2.6\%) which is in accordance with the finding of Rampal et al (2002), Kour (2004), Rampal et al. (2005), Gupta et al. (2008), Jaswal (2008) and Kewal (2010) who also recorded highest percentage of biodegradable waste.

When a comparative study of solid waste generated at all the four study zones was made, it was observed that the total average solid waste generated (Kg/capita/month) was found to be maximum in Zone-IV $(71.961 \pm 37.476 \mathrm{Kg})$ followed by Zone- I $(28.735 \pm 8.866 \mathrm{Kg}), \quad$ Zone-III $(19.396 \pm 4.777 \mathrm{Kg})$ and Zone-II $(19.039 \pm 5.364$ $\mathrm{Kg})$. The minimum value $(19.039 \pm 5.364 \mathrm{Kg})$ of waste generated was exhibited by Zone -II.Though the results have shown a variation in the solid waste generation in the study area during different months of the year, but no set pattern of waste generation was observed. From the studies, it has been observed that people don't dispose off the waste properly in the area rather throw it in open or vacant plots, on roadsides, streets or nallahs. Moreover, for the final disposal of wastes, open dump method is generally followed by the municipality. There are mainly two dump sites in the area. One is near the river Chenab and another in the outskirts of Akhnoor Town.

Thus on the basis of studies conducted in Akhnoor town, the following conclusions have been drawn:

1. The Municipal solid waste of Akhnoor town is highly heterogeneous in nature with high percentage of biodegradable material.

2. Although maximum percentage of solid waste generated within the municipal limits of Akhnoor town is biodegradable and organic in nature but its decay within the study area provide breeding ground for a no. of pathogens such a flies, mosquitoes, rodents etc. thereby posing threats to the health of the people residing in the area. 
Table 1:- Qualitative and Quantitative Composition of Solid Waste (Kg/Capita/Month) in H.1-H.5 at Zone-I

\begin{tabular}{|c|c|c|c|c|c|c|c|c|c|c|c|c|c|c|c|c|c|c|c|c|}
\hline \multicolumn{21}{|c|}{ Houses Zone I } \\
\hline $\begin{array}{l}\text { No. of family } \\
\text { members }\end{array}$ & \multicolumn{4}{|c|}{5} & \multicolumn{4}{|c|}{4} & \multicolumn{4}{|c|}{4} & \multicolumn{4}{|c|}{6} & \multicolumn{4}{|c|}{4} \\
\hline \multirow[b]{2}{*}{ Months } & \multicolumn{4}{|c|}{ H.1 } & \multicolumn{4}{|c|}{ H. 2} & \multicolumn{4}{|c|}{ H. 3} & \multicolumn{4}{|c|}{ H.4 } & \multicolumn{4}{|c|}{ H.5 } \\
\hline & $\begin{array}{c}\text { Bio } \\
\text { Degrada } \\
\text { ble waste }\end{array}$ & $\begin{array}{c}\text { Non } \\
\text { Bio } \\
\text { Deg. } \\
\text { waste }\end{array}$ & $\begin{array}{l}\text { Inert } \\
\text { Solid } \\
\text { Waste }\end{array}$ & $\begin{array}{l}\text { Total } \\
\text { Solid } \\
\text { waste }\end{array}$ & $\begin{array}{c}\text { Bio } \\
\text { Deg. } \\
\text { waste }\end{array}$ & $\begin{array}{c}\text { Non } \\
\text { Bio } \\
\text { Deg. } \\
\text { waste }\end{array}$ & $\begin{array}{l}\text { Inert } \\
\text { Solid } \\
\text { Waste }\end{array}$ & $\begin{array}{l}\text { Total } \\
\text { Solid } \\
\text { waste }\end{array}$ & $\begin{array}{c}\text { Bio } \\
\text { Deg. } \\
\text { waste }\end{array}$ & $\begin{array}{c}\text { Non } \\
\text { Bio } \\
\text { Deg. } \\
\text { waste }\end{array}$ & $\begin{array}{c}\text { Inert } \\
\text { Solid } \\
\text { Waste }\end{array}$ & $\begin{array}{l}\text { Total } \\
\text { Solid } \\
\text { waste }\end{array}$ & $\begin{array}{c}\text { Bio } \\
\text { Deg. } \\
\text { waste }\end{array}$ & $\begin{array}{l}\text { Non Bio } \\
\text { Deg. } \\
\text { waste }\end{array}$ & $\begin{array}{c}\text { Inert } \\
\text { Solid } \\
\text { Waste }\end{array}$ & $\begin{array}{l}\text { Total } \\
\text { Solid } \\
\text { waste }\end{array}$ & $\begin{array}{c}\text { Bio Deg. } \\
\text { waste }\end{array}$ & $\begin{array}{c}\text { Non } \\
\text { Bio } \\
\text { Deg. } \\
\text { waste }\end{array}$ & $\begin{array}{l}\text { Inert } \\
\text { Solid } \\
\text { Waste }\end{array}$ & $\begin{array}{l}\text { Total } \\
\text { Solid } \\
\text { waste }\end{array}$ \\
\hline June & 29.850 & 2.250 & 0.600 & 32.700 & 18.900 & 0.750 & 1.200 & 20.850 & 13.980 & 0.300 & 1.050 & 15.330 & 48.600 & 5.400 & 2.100 & 56.100 & 4.500 & 0.300 & 0.750 & 5.550 \\
\hline July & 36.060 & 7.830 & 0.780 & 44.670 & 14.880 & 3.600 & 0.960 & 19.440 & 20.820 & 2.730 & 0.390 & 23.940 & 53.400 & 6.240 & 0.360 & 60.000 & 10.200 & 0.930 & 0.480 & 11.610 \\
\hline August & 46.710 & 6.930 & 1.140 & 54.780 & 39.420 & 0.870 & 0.780 & 41.070 & 18.480 & 1.560 & 0.960 & 21.000 & 56.460 & 3.690 & 0.630 & 60.780 & 35.220 & 1.830 & 0.540 & 37.590 \\
\hline September & 44.400 & 2.490 & 0.600 & 47.490 & 13.530 & 2.040 & 1.050 & 16.620 & 18.420 & 3.210 & 0.750 & 22.380 & 49.830 & 3.690 & 0.660 & 54.180 & 11.640 & 0.660 & 0.630 & 12.930 \\
\hline October & 37.590 & 3.360 & 0.420 & 41.370 & 11.250 & 0.630 & 0.390 & 12.270 & 13.830 & 1.620 & 0.300 & 15.750 & 37.470 & 2.850 & 0.720 & 41.040 & 11.640 & 0.570 & 0.420 & 12.630 \\
\hline November & 33.660 & 2.190 & 0.360 & 36.210 & 10.320 & 0.570 & 0.300 & 11.190 & 10.350 & 1.560 & 0.270 & 12.180 & 36.300 & 2.520 & 0.600 & 39.420 & 11.010 & 0.750 & 0.360 & 12.120 \\
\hline December & 43.320 & 5.640 & 0.930 & 49.890 & 14.490 & 0.540 & 0.840 & 15.870 & 15.990 & 3.210 & 0.540 & 19.740 & 50.130 & 3.090 & 0.870 & 54.090 & 12.210 & 0.840 & 0.780 & 13.830 \\
\hline January & 46.710 & 5.820 & 0.360 & 52.890 & 18.990 & 1.380 & 0.480 & 20.850 & 11.160 & 1.620 & 0.330 & 13.110 & 37.980 & 3.060 & 0.840 & 41.880 & 11.460 & 1.980 & 0.360 & 13.800 \\
\hline February & 36.480 & 3.540 & 0.870 & 40.890 & 13.650 & 1.050 & 0.540 & 15.240 & 11.010 & 1.740 & 0.390 & 13.140 & 31.920 & 4.140 & 0.540 & 36.600 & 8.430 & 0.480 & 0.450 & 9.360 \\
\hline March & 32.760 & 5.700 & 1.260 & 39.720 & 14.700 & 2.130 & 1.140 & 17.970 & 12.600 & 3.630 & 1.080 & 17.310 & 31.590 & 4.140 & 1.140 & 36.870 & 13.980 & 2.400 & 0.870 & 17.250 \\
\hline April & 40.560 & 4.860 & 1.530 & 46.950 & 21.240 & 2.220 & 1.230 & 24.690 & 14.130 & 2.850 & 1.170 & 18.150 & 39.630 & 4.680 & 1.170 & 45.480 & 16.890 & 1.770 & 0.930 & 19.590 \\
\hline May & 27.480 & 2.760 & 0.960 & 31.200 & 15.030 & 2.010 & 1.170 & 18.210 & 33.120 & 0.420 & 1.110 & 34.650 & 42.090 & 4.050 & 0.570 & 46.710 & 3.750 & 0.390 & 0.840 & 4.980 \\
\hline Total & 455.580 & 53.370 & 9.810 & 518.760 & 206.400 & 17.790 & 10.080 & 234.270 & $\begin{array}{c}193.89 \\
0\end{array}$ & 24.450 & 8.340 & 226.680 & $\begin{array}{c}515.40 \\
0\end{array}$ & 47.550 & 10.200 & 573.150 & 150.930 & $\begin{array}{c}12.90 \\
0\end{array}$ & 7.410 & 171.240 \\
\hline $\begin{array}{l}\text { Total per } \\
\text { month }\end{array}$ & 37.965 & 4.448 & 0.818 & $\begin{array}{c}15562.80 \\
0\end{array}$ & 17.200 & 1.483 & 0.840 & $\begin{array}{c}7028.10 \\
0\end{array}$ & 16.158 & 2.038 & 0.695 & 6800.40 & 42.950 & 3.963 & 0.850 & 17194.5 & 12.578 & 1.075 & 0.618 & 5137.200 \\
\hline $\begin{array}{l}\text { Per Month/ } \\
\text { capita }\end{array}$ & 7.593 & 0.890 & 0.164 & 3112.560 & 4.300 & 0.371 & 0.210 & $\begin{array}{l}1757.02 \\
5\end{array}$ & 4.039 & 0.509 & 0.174 & 1700.10 & 7.158 & 0.660 & 0.142 & 2865.75 & 3.144 & 0.269 & 0.154 & 1284.300 \\
\hline Total per day & 1.266 & 0.148 & 0.027 & 103.752 & 0.573 & 0.049 & 0.028 & 58.568 & 0.539 & 0.068 & 0.023 & 56.670 & 1.432 & 0.132 & 0.028 & 95.525 & 0.419 & 0.036 & 0.021 & 42.810 \\
\hline $\begin{array}{c}\text { Per day/ } \\
\text { capita }\end{array}$ & 0.253 & 0.030 & 0.005 & 1296.900 & 0.143 & 0.012 & 0.007 & 585.675 & 0.135 & 0.017 & 0.006 & 566.700 & 0.239 & 0.022 & 0.005 & 1432.875 & 0.105 & 0.009 & 0.005 & 428.100 \\
\hline Average & 37.965 & 4.448 & 0.818 & 7.576 & 17.200 & 1.483 & 0.840 & 7.751 & 16.158 & 2.038 & 0.695 & 6.240 & 42.950 & 3.963 & 0.850 & 8.888 & 12.578 & 1.075 & 0.618 & 8.443 \\
\hline Std. Dev. & 6.447 & 1.937 & 0.371 & 8.755 & 7.679 & 0.934 & 0.338 & 8.951 & 6.268 & 1.085 & 0.360 & 7.713 & 8.442 & 1.082 & 0.460 & 9.984 & 8.005 & 0.717 & 0.012 & 8.734 \\
\hline
\end{tabular}

A.V. -Average

S.D.- Standard Deviation 
Sharma and Gupta

Table2 :- Qualitative and Quantitative Composition of Solid Waste (Kg/Capita/Month) in H.1-H.5 at Zone-II

\begin{tabular}{|c|c|c|c|c|c|c|c|c|c|c|c|c|c|c|c|c|c|c|c|c|}
\hline \multicolumn{21}{|c|}{ Houses Zone II } \\
\hline $\begin{array}{l}\text { No. of family } \\
\text { members }\end{array}$ & & & & & & & & & & & & & & & & & & & & \\
\hline \multirow[b]{2}{*}{ Months } & \multicolumn{4}{|c|}{ H.1 } & \multicolumn{4}{|c|}{ H.2 } & \multicolumn{4}{|c|}{ H.3 } & \multicolumn{4}{|c|}{ H.4 } & \multicolumn{4}{|c|}{ H.5 } \\
\hline & $\begin{array}{c}\text { Bio } \\
\text { Degrada } \\
\text { ble } \\
\text { waste } \\
\end{array}$ & $\begin{array}{c}\text { Non } \\
\text { Bio } \\
\text { Degrad } \\
\text { able } \\
\text { waste } \\
\end{array}$ & $\begin{array}{l}\text { Inert } \\
\text { Solid } \\
\text { Waste }\end{array}$ & $\begin{array}{l}\text { Total } \\
\text { Solid } \\
\text { waste }\end{array}$ & $\begin{array}{c}\text { Bio } \\
\text { Degrad } \\
\text { able } \\
\text { waste }\end{array}$ & $\begin{array}{l}\text { Non Bio } \\
\text { Degrada } \\
\text { ble waste }\end{array}$ & $\begin{array}{c}\text { Inert } \\
\text { Solid } \\
\text { Waste }\end{array}$ & $\begin{array}{l}\text { Total } \\
\text { Solid } \\
\text { waste }\end{array}$ & $\begin{array}{c}\text { Bio } \\
\text { Degrada } \\
\text { ble waste }\end{array}$ & $\begin{array}{c}\text { Non } \\
\text { Bio } \\
\text { Degrad } \\
\text { able } \\
\text { waste } \\
\end{array}$ & $\begin{array}{l}\text { Inert } \\
\text { Solid } \\
\text { Waste }\end{array}$ & $\begin{array}{l}\text { Total } \\
\text { Solid } \\
\text { waste }\end{array}$ & $\begin{array}{c}\text { Bio } \\
\text { Degra } \\
\text { dable } \\
\text { waste } \\
\end{array}$ & $\begin{array}{c}\text { Non } \\
\text { Bio } \\
\text { Degrad } \\
\text { able } \\
\text { waste } \\
\end{array}$ & $\begin{array}{l}\text { Inert } \\
\text { Solid } \\
\text { Waste }\end{array}$ & $\begin{array}{l}\text { Total } \\
\text { Solid } \\
\text { waste }\end{array}$ & $\begin{array}{c}\text { Bio } \\
\text { Degrad } \\
\text { able } \\
\text { waste } \\
\end{array}$ & $\begin{array}{c}\text { Non } \\
\text { Bio } \\
\text { Degra } \\
\text { dable } \\
\text { waste } \\
\end{array}$ & $\begin{array}{l}\text { Inert } \\
\text { Solid } \\
\text { Waste }\end{array}$ & $\begin{array}{l}\text { Total } \\
\text { Solid } \\
\text { waste }\end{array}$ \\
\hline June & 2.310 & 0.300 & 0.150 & 2.760 & 29.700 & 6.150 & 0.540 & 36.390 & 3.750 & 0.600 & 0.630 & 4.980 & 23.70 & 7.170 & 0.810 & 31.680 & 17.400 & 2.400 & 1.020 & 20.820 \\
\hline July & 5.820 & 1.740 & 0.600 & 8.160 & 25.680 & 7.470 & 0.630 & 33.780 & 5.340 & 0.600 & 0.300 & 6.240 & 27.36 & 2.190 & 0.690 & 30.240 & 20.580 & 6.060 & 1.140 & 27.780 \\
\hline August & 4.080 & 0.900 & 0.390 & 5.370 & 29.310 & 4.260 & 0.600 & 34.170 & 5.040 & 1.830 & 0.540 & 7.410 & 23.73 & 4.380 & 0.900 & 29.010 & 16.560 & 4.050 & 0.840 & 21.450 \\
\hline September & 3.630 & 0.720 & 0.540 & 4.890 & 23.700 & 3.180 & 0.480 & 27.360 & 5.430 & 1.260 & 0.360 & 7.050 & 18.18 & 4.110 & 0.690 & 22.980 & 16.080 & 2.910 & 0.930 & 19.920 \\
\hline October & 7.020 & 2.100 & 0.390 & 9.510 & 22.110 & 4.710 & 0.720 & 27.540 & 11.160 & 2.880 & 0.600 & 14.640 & 21.06 & 3.180 & 0.750 & 24.990 & 26.310 & 5.610 & 0.870 & 32.790 \\
\hline November & 6.630 & 1.440 & 0.270 & 8.340 & 20.700 & 4.470 & 0.570 & 25.740 & 9.900 & 2.550 & 0.540 & 12.990 & 19.23 & 3.090 & 0.600 & 22.920 & 24.870 & 4.350 & 0.810 & 30.030 \\
\hline December & 4.320 & 0.720 & 0.330 & 5.370 & 25.800 & 2.820 & 0.600 & 29.220 & 9.240 & 2.040 & 0.630 & 11.910 & 26.16 & 4.650 & 1.050 & 31.860 & 24.660 & 4.590 & 0.870 & 30.120 \\
\hline January & 7.260 & 2.100 & 0.300 & 9.660 & 21.720 & 4.440 & 0.780 & 26.940 & 12.330 & 1.560 & 0.540 & 14.430 & 23.55 & 2.730 & 0.870 & 27.150 & 26.580 & 5.490 & 0.930 & 33.000 \\
\hline February & 5.400 & 1.680 & 0.390 & 7.470 & 15.720 & 3.990 & 0.630 & 20.340 & 8.910 & 0.450 & 0.300 & 9.660 & 20.97 & 5.160 & 0.570 & 26.700 & 22.200 & 3.300 & 0.840 & 26.340 \\
\hline March & 5.580 & 0.870 & 0.570 & 7.020 & 9.540 & 7.620 & 0.840 & 18.000 & 7.440 & 3.360 & 0.600 & 11.400 & 20.79 & 9.030 & 1.140 & 30.960 & 20.010 & 7.770 & 1.230 & 29.010 \\
\hline April & 1.470 & 0.150 & 0.240 & 1.860 & 19.770 & 3.510 & 0.750 & 24.030 & 3.840 & 0.630 & 0.930 & 5.400 & 16.20 & 3.930 & 0.870 & 21.000 & 12.000 & 4.590 & 1.170 & 17.760 \\
\hline May & 2.070 & 0.270 & 0.180 & 2.520 & 22.530 & 3.630 & 0.600 & 26.760 & 3.510 & 0.570 & 0.870 & 4.950 & 19.56 & 4.650 & 0.810 & 25.020 & 11.430 & 2.190 & 0.930 & 14.550 \\
\hline Total & 55.590 & 12.990 & 4.350 & 72.930 & 266.280 & 56.250 & 7.740 & 330.270 & 85.890 & 18.330 & 6.840 & 111.060 & $\begin{array}{c}260.4 \\
9 \\
\end{array}$ & 54.270 & 9.750 & 324.510 & 238.680 & 53.31 & 11.580 & 303.570 \\
\hline $\begin{array}{l}\text { Total per } \\
\text { month }\end{array}$ & 4.633 & 1.083 & 0.363 & $\begin{array}{c}2187.90 \\
0\end{array}$ & 22.190 & 4.688 & 0.645 & $\begin{array}{c}9908.10 \\
0\end{array}$ & 7.158 & 1.528 & 0.570 & 3331.80 & $\begin{array}{c}21.70 \\
8\end{array}$ & 4.523 & 0.813 & 9735.30 & 19.890 & 4.443 & 0.965 & $\begin{array}{c}9107.10 \\
0\end{array}$ \\
\hline $\begin{array}{l}\text { Per Month/ } \\
\text { capita }\end{array}$ & 2.316 & 0.541 & 0.181 & $\begin{array}{c}1093.95 \\
0\end{array}$ & 5.548 & 1.172 & 0.161 & $\begin{array}{c}2477.02 \\
5\end{array}$ & 2.386 & 0.509 & 0.190 & 1110.60 & 4.342 & 0.905 & 0.163 & 1947.06 & 3.978 & 0.889 & 0.193 & $\begin{array}{c}1821.42 \\
0\end{array}$ \\
\hline Total per day & 0.154 & 0.036 & 0.012 & 36.465 & 0.740 & 0.156 & 0.022 & 82.568 & 0.239 & 0.051 & 0.019 & 37.020 & 0.724 & 0.151 & 0.027 & 64.902 & 0.663 & 0.148 & 0.032 & 60.714 \\
\hline Per day/ capita & 0.077 & 0.018 & 0.006 & 182.325 & 0.185 & 0.039 & 0.005 & 825.675 & 0.080 & 0.017 & 0.006 & 277.650 & 0.145 & 0.030 & 0.005 & 811.275 & 0.133 & 0.030 & 0.006 & 758.925 \\
\hline Average & 4.633 & 1.083 & 0.363 & 2.710 & 22.190 & 4.688 & 0.645 & 5.423 & 7.158 & 1.528 & 0.570 & 3.700 & 21.70 & 4.523 & 0.813 & 3.725 & 19.890 & 4.443 & 0.965 & 6.166 \\
\hline Std. Dev. & 1.974 & 0.705 & 0.148 & 2.827 & 5.599 & 1.582 & 0.106 & 7.287 & 3.082 & 1.012 & 0.196 & 4.289 & 3.283 & 1.926 & 0.169 & 5.379 & 5.266 & 1.631 & 0.142 & 7.039 \\
\hline
\end{tabular}

A.V. -Average

S.D.- Standard Deviation

4

Environment Conservation Journal

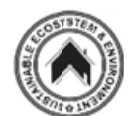


Table3 :- Qualitative and Quantitative Composition of Solid Waste (Kg/Capita/Month) in H.1-H.5 at Zone-III

\begin{tabular}{|c|c|c|c|c|c|c|c|c|c|c|c|c|c|c|c|c|c|c|c|c|}
\hline \multicolumn{21}{|c|}{ Houses Zone III } \\
\hline $\begin{array}{l}\text { No. of } \\
\text { family }\end{array}$ & \multicolumn{4}{|c|}{5} & \multicolumn{4}{|c|}{4} & \multicolumn{4}{|c|}{4} & \multicolumn{4}{|c|}{4} & \multicolumn{4}{|c|}{6} \\
\hline & \multicolumn{4}{|c|}{ H.1 } & \multicolumn{4}{|c|}{ H. 2} & \multicolumn{4}{|c|}{ H. 3} & \multicolumn{4}{|c|}{ H.4 } & \multicolumn{4}{|c|}{ H.5 } \\
\hline Months & $\begin{array}{c}\text { Bio } \\
\text { Degra } \\
\text { dable } \\
\text { waste }\end{array}$ & $\begin{array}{l}\text { Non Bio } \\
\text { Degrada } \\
\text { ble waste }\end{array}$ & $\begin{array}{l}\text { Inert } \\
\text { Solid } \\
\text { Waste }\end{array}$ & $\begin{array}{c}\text { Total Solid } \\
\text { waste }\end{array}$ & $\begin{array}{c}\text { Bio } \\
\text { Degrad } \\
\text { able } \\
\text { waste } \\
\end{array}$ & $\begin{array}{c}\text { Non } \\
\text { Bio } \\
\text { Degra } \\
\text { dable } \\
\text { waste } \\
\end{array}$ & $\begin{array}{l}\text { Inert } \\
\text { Solid } \\
\text { Waste }\end{array}$ & $\begin{array}{l}\text { Total } \\
\text { Solid } \\
\text { waste }\end{array}$ & $\begin{array}{c}\text { Bio } \\
\text { Degrad } \\
\text { able } \\
\text { waste }\end{array}$ & $\begin{array}{l}\text { Non Bio } \\
\text { Degrada } \\
\text { ble waste }\end{array}$ & $\begin{array}{l}\text { Inert } \\
\text { Solid } \\
\text { Waste }\end{array}$ & $\begin{array}{l}\text { Total } \\
\text { Solid } \\
\text { waste }\end{array}$ & $\begin{array}{c}\text { Bio } \\
\text { Degrad } \\
\text { able } \\
\text { waste } \\
\end{array}$ & $\begin{array}{l}\text { Non Bio } \\
\text { Degrada } \\
\text { ble waste }\end{array}$ & $\begin{array}{l}\text { Inert } \\
\text { Solid } \\
\text { Waste }\end{array}$ & $\begin{array}{l}\text { Total } \\
\text { Solid } \\
\text { waste }\end{array}$ & $\begin{array}{c}\text { Bio } \\
\text { Degrad } \\
\text { able } \\
\text { waste }\end{array}$ & $\begin{array}{c}\text { Non } \\
\text { Bio } \\
\text { Degra } \\
\text { dable } \\
\text { waste } \\
\end{array}$ & $\begin{array}{l}\text { Inert } \\
\text { Solid } \\
\text { Waste }\end{array}$ & $\begin{array}{l}\text { Total } \\
\text { Solid } \\
\text { waste }\end{array}$ \\
\hline June & $\begin{array}{c}18.48 \\
0 \\
\end{array}$ & 6.450 & 1.320 & 26.250 & 20.310 & 7.530 & 0.930 & 28.770 & 12.870 & 6.630 & 0.810 & 20.310 & 19.590 & 5.850 & 1.560 & 27.000 & 24.900 & 2.040 & 0.360 & 27.300 \\
\hline July & $\begin{array}{c}19.44 \\
0\end{array}$ & 4.920 & 1.050 & 25.410 & 11.850 & 2.250 & 0.690 & 14.790 & 12.330 & 1.110 & 0.930 & 14.370 & 19.800 & 3.870 & 1.980 & 25.650 & 24.300 & 3.900 & 0.450 & 28.650 \\
\hline August & $\begin{array}{c}13.50 \\
0 \\
\end{array}$ & 5.250 & 1.230 & 19.980 & 12.090 & 5.250 & 0.900 & 18.240 & 7.740 & 1.170 & 0.600 & 9.510 & 15.780 & 5.520 & 1.470 & 22.770 & 24.030 & 4.290 & 0.630 & 28.950 \\
\hline September & $\begin{array}{c}14.73 \\
0\end{array}$ & 2.670 & 0.870 & 18.270 & 12.540 & 1.590 & 0.750 & 14.880 & 10.260 & 0.960 & 0.660 & 11.880 & 15.450 & 2.490 & 1.980 & 19.920 & 22.680 & 4.560 & 1.170 & 28.410 \\
\hline October & $\begin{array}{c}14.70 \\
0\end{array}$ & 2.280 & 0.630 & 17.610 & 12.660 & 1.380 & 0.540 & 14.580 & 10.800 & 0.870 & 0.600 & 12.270 & 17.520 & 2.430 & 1.560 & 21.510 & 21.810 & 2.130 & 1.020 & 24.960 \\
\hline November & $\begin{array}{c}14.01 \\
0\end{array}$ & 1.470 & 0.420 & 15.900 & 11.460 & 1.080 & 0.510 & 13.050 & 10.320 & 0.690 & 0.450 & 11.460 & 15.660 & 2.100 & 0.750 & 18.510 & 19.470 & 2.220 & 0.870 & 22.560 \\
\hline December & $\begin{array}{c}15.96 \\
0\end{array}$ & 3.660 & 0.960 & 20.580 & 11.790 & 4.590 & 1.260 & 17.640 & 7.560 & 2.340 & 0.750 & 10.650 & 13.530 & 3.690 & 1.230 & 18.450 & 20.100 & 7.410 & 0.570 & 28.080 \\
\hline January & $\begin{array}{c}16.65 \\
0 \\
\end{array}$ & 4.620 & 0.930 & 22.200 & 14.340 & 6.570 & 0.570 & 21.480 & 14.850 & 4.530 & 0.600 & 19.980 & 13.530 & 4.320 & 1.050 & 18.900 & 20.070 & 4.830 & 0.270 & 25.170 \\
\hline February & $\begin{array}{c}13.59 \\
0\end{array}$ & 1.830 & 0.540 & 15.960 & 13.110 & 2.010 & 0.600 & 15.720 & 12.330 & 2.640 & 0.630 & 15.600 & 10.260 & 3.330 & 0.960 & 14.550 & 20.490 & 4.920 & 0.300 & 25.710 \\
\hline March & $\begin{array}{c}11.94 \\
0 \\
\end{array}$ & 5.220 & 1.470 & 18.630 & 10.560 & 4.830 & 1.530 & 16.920 & 12.300 & 3.540 & 1.260 & 17.100 & 9.690 & 4.530 & 2.130 & 16.350 & 14.850 & 6.150 & 1.260 & 22.260 \\
\hline April & $\begin{array}{c}11.79 \\
0\end{array}$ & 4.500 & 1.260 & 17.550 & 11.760 & 3.660 & 1.380 & 16.800 & 11.640 & 3.870 & 1.170 & 16.680 & 13.230 & 4.680 & 0.840 & 18.750 & 17.520 & 1.800 & 1.590 & 20.910 \\
\hline May & $\begin{array}{c}13.32 \\
0\end{array}$ & 3.480 & 1.200 & 18.000 & 12.990 & 3.210 & 1.230 & 17.430 & 8.130 & 3.960 & 0.840 & 12.930 & 12.120 & 5.220 & 1.440 & 18.780 & 18.000 & 1.740 & 0.540 & 20.280 \\
\hline Total & $\begin{array}{c}178.1 \\
10 \\
\end{array}$ & 46.350 & 11.880 & 236.340 & 155.460 & $\begin{array}{c}43.95 \\
0 \\
\end{array}$ & $\begin{array}{c}10.89 \\
0 \\
\end{array}$ & 210.300 & 131.130 & 32.310 & 9.300 & 172.740 & 176.160 & 48.030 & 16.950 & 241.140 & 248.220 & $\begin{array}{c}45.99 \\
0 \\
\end{array}$ & 9.030 & 303.240 \\
\hline $\begin{array}{l}\text { Total per } \\
\text { month }\end{array}$ & $\begin{array}{c}14.84 \\
3\end{array}$ & 3.863 & 0.990 & 7090.20 & 12.955 & 3.663 & 0.908 & 6309.00 & 10.928 & 2.693 & 0.775 & 5182.200 & 14.680 & 4.003 & 1.413 & 7234.20 & 20.685 & 3.833 & 0.753 & 9097.20 \\
\hline $\begin{array}{c}\text { Per } \\
\text { Month/ } \\
\text { capita }\end{array}$ & 2.969 & 0.773 & 0.198 & 1418.04 & 3.239 & 0.916 & 0.227 & 1577.25 & 2.732 & 0.673 & 0.194 & 1295.550 & 3.670 & 1.001 & 0.353 & 1808.55 & 3.448 & 0.639 & 0.125 & 1516.20 \\
\hline $\begin{array}{c}\begin{array}{c}\text { Total per } \\
\text { day }\end{array} \\
\end{array}$ & 0.495 & 0.129 & 0.033 & 47.268 & 0.432 & 0.122 & 0.030 & 52.575 & 0.364 & 0.090 & 0.026 & 43.185 & 0.489 & 0.133 & 0.047 & 60.285 & 0.690 & 0.128 & 0.025 & 50.540 \\
\hline $\begin{array}{c}\text { Per day/ } \\
\text { capita }\end{array}$ & 0.099 & 0.026 & 0.007 & 590.850 & 0.108 & 0.031 & 0.008 & 525.750 & 0.091 & 0.022 & 0.006 & 431.850 & 0.122 & 0.033 & 0.012 & 602.850 & 0.115 & 0.021 & 0.004 & 758.100 \\
\hline Average & $\begin{array}{c}14.84 \\
3 \\
\end{array}$ & 3.863 & 0.990 & 3.386 & 12.955 & 3.663 & 0.908 & 4.156 & 10.928 & 2.693 & 0.775 & 3.563 & 14.680 & 4.003 & 1.413 & 3.602 & 20.685 & 3.833 & 0.753 & 3.126 \\
\hline Std. Dev. & 2.394 & 1.553 & 0.329 & 4.276 & 2.505 & 2.116 & 0.358 & 4.980 & 2.249 & 1.856 & 0.244 & 4.348 & 3.260 & 1.243 & 0.461 & 4.964 & 3.027 & 1.866 & 0.426 & 5.319 \\
\hline
\end{tabular}

A.V. -Average

S.D.- Standard Deviation 
Sharma and Gupta

Table4 :- Qualitative and Quantitative Composition of Solid Waste (Kg/Capita/Month) in H.1-H.5 at Zone-IV

\begin{tabular}{|c|c|c|c|c|c|c|c|c|c|c|c|c|c|c|c|c|c|c|c|c|}
\hline \multirow{3}{*}{\begin{tabular}{|c|}
$\begin{array}{l}\text { No. of } \\
\text { family } \\
\text { members }\end{array}$ \\
\\
Months \\
\end{tabular}} & \multicolumn{4}{|c|}{4} & \multicolumn{4}{|c|}{4} & \multicolumn{4}{|c|}{5} & \multicolumn{4}{|c|}{4} & \multicolumn{4}{|c|}{3} \\
\hline & \multicolumn{4}{|c|}{ H.1 } & \multicolumn{4}{|c|}{ H. 2} & \multicolumn{4}{|c|}{ H.3 } & \multicolumn{4}{|c|}{ H.4 } & \multicolumn{4}{|c|}{ H.5 } \\
\hline & $\begin{array}{c}\text { Bio } \\
\text { Degrada } \\
\text { ble waste }\end{array}$ & $\begin{array}{l}\text { Non Bio } \\
\text { Degrada } \\
\text { ble waste }\end{array}$ & $\begin{array}{l}\text { Inert } \\
\text { Solid } \\
\text { Waste }\end{array}$ & $\begin{array}{l}\text { Total } \\
\text { Solid } \\
\text { waste }\end{array}$ & $\begin{array}{c}\text { Bio } \\
\text { Degrad } \\
\text { able } \\
\text { waste }\end{array}$ & 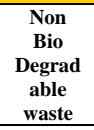 & $\begin{array}{l}\text { Inert } \\
\text { Solid } \\
\text { Waste }\end{array}$ & $\begin{array}{l}\text { Total } \\
\text { Solid } \\
\text { waste }\end{array}$ & $\begin{array}{c}\text { Bio } \\
\text { Degrad } \\
\text { able } \\
\text { waste }\end{array}$ & $\begin{array}{c}\text { Non Bio } \\
\text { Degradable } \\
\text { waste }\end{array}$ & $\begin{array}{l}\text { Inert } \\
\text { Solid } \\
\text { Waste }\end{array}$ & $\begin{array}{l}\text { Total } \\
\text { Solid } \\
\text { waste }\end{array}$ & $\begin{array}{c}\text { Bio } \\
\text { Degradable } \\
\text { waste }\end{array}$ & $\begin{array}{l}\text { Non Bio } \\
\text { Degrada } \\
\text { ble waste }\end{array}$ & $\begin{array}{l}\text { Inert } \\
\text { Solid } \\
\text { Waste }\end{array}$ & $\begin{array}{l}\text { Total } \\
\text { Solid } \\
\text { waste }\end{array}$ & $\begin{array}{c}\text { Bio } \\
\text { Degrad } \\
\text { able } \\
\text { waste }\end{array}$ & $\begin{array}{c}\text { Non } \\
\text { Bio } \\
\text { Degrad } \\
\text { able } \\
\text { waste } \\
\end{array}$ & $\begin{array}{l}\text { Inert } \\
\text { Solid } \\
\text { Waste }\end{array}$ & $\begin{array}{l}\text { Total } \\
\text { Solid } \\
\text { waste }\end{array}$ \\
\hline June & 193.500 & 6.360 & 1.200 & 201.060 & 155.970 & 3.000 & 0.750 & 159.720 & 93.300 & 7.830 & 1.500 & 102.630 & 75.960 & 5.820 & 0.450 & 82.230 & 25.980 & 0.300 & 1.050 & 27.330 \\
\hline July & 165.450 & 61.800 & 1.950 & 229.200 & 131.160 & 53.520 & 1.860 & 186.540 & 100.620 & 6.090 & 1.350 & 108.060 & 54.930 & 3.090 & 1.800 & 59.820 & 4.860 & 2.790 & 1.080 & 8.730 \\
\hline August & 107.760 & 30.510 & 1.560 & 139.830 & 86.550 & 33.510 & 2.070 & 122.130 & 103.830 & 19.320 & 1.440 & 124.590 & 51.930 & 8.130 & 1.680 & 61.740 & 7.080 & 0.090 & 0.750 & 7.920 \\
\hline September & 111.960 & 40.410 & 1.830 & 154.200 & 85.950 & 28.680 & 1.560 & 116.190 & 99.300 & 5.790 & 1.170 & 106.260 & 53.400 & 12.120 & 1.530 & 67.050 & 4.380 & 0.660 & 0.600 & 5.640 \\
\hline October & 56.580 & 43.650 & 1.290 & 101.520 & 35.700 & 23.610 & 1.470 & 60.780 & 76.890 & 23.400 & 1.080 & 101.370 & 34.830 & 14.220 & 1.170 & 50.220 & 4.530 & 0.540 & 0.540 & 5.610 \\
\hline November & 48.420 & 47.520 & 1.140 & 97.080 & 34.620 & 30.180 & 1.230 & 66.030 & 71.850 & 23.520 & 0.930 & 96.300 & 33.720 & 14.280 & 0.840 & 48.840 & 4.530 & 1.230 & 0.420 & 6.180 \\
\hline December & 81.840 & 13.650 & 1.260 & 96.750 & 72.240 & 7.020 & 1.470 & 80.730 & 72.210 & 7.020 & 1.350 & 80.580 & 60.690 & 9.540 & 0.750 & 70.980 & 10.980 & 0.600 & 0.930 & 12.510 \\
\hline January & 67.650 & 11.730 & 1.560 & 80.940 & 36.780 & 10.020 & 1.440 & 48.240 & 67.440 & 7.710 & 1.380 & 76.530 & 31.230 & 5.400 & 0.870 & 37.500 & 8.550 & 0.900 & 1.170 & 10.620 \\
\hline February & 46.860 & 7.890 & 1.440 & 56.190 & 38.370 & 7.380 & 1.230 & 46.980 & 32.430 & 8.760 & 1.140 & 42.330 & 23.430 & 4.650 & 0.600 & 28.680 & 7.500 & 1.050 & 0.930 & 9.480 \\
\hline March & 35.640 & 8.610 & 0.960 & 45.210 & 29.820 & 5.250 & 1.200 & 36.270 & 26.850 & 6.930 & 0.570 & 34.350 & 24.720 & 2.760 & 0.450 & 27.930 & 8.790 & 0.960 & 0.750 & 10.500 \\
\hline April & 75.510 & 6.240 & 1.380 & 83.130 & 60.900 & 4.110 & 0.870 & 65.880 & 73.140 & 7.110 & 1.740 & 81.990 & 60.480 & 5.790 & 0.750 & 67.020 & 17.910 & 0.450 & 1.140 & 19.500 \\
\hline May & 99.690 & 5.160 & 1.260 & 106.110 & 96.390 & 2.940 & 0.780 & 100.110 & 68.310 & 4.620 & 1.530 & 74.460 & 59.790 & 3.810 & 0.570 & 64.170 & 15.510 & 0.570 & 1.080 & 17.160 \\
\hline Total & 1090.860 & 283.530 & 16.83 & 1391.22 & 864.450 & 209.220 & 15.93 & 1089.60 & 886.170 & 128.100 & 15.180 & 1029.45 & 565.110 & 89.610 & 11.46 & 666.180 & 120.600 & 10.140 & $\begin{array}{c}10.44 \\
0\end{array}$ & 141.180 \\
\hline $\begin{array}{c}\begin{array}{c}\text { Total per } \\
\text { month }\end{array} \\
\end{array}$ & 90.905 & 23.628 & 1.403 & 41736.6 & 72.038 & 17.435 & 1.328 & 32688.0 & 73.848 & 10.675 & 1.265 & 30883.5 & 47.093 & 7.468 & 0.955 & 19985.4 & 10.050 & 0.845 & 0.870 & 4235.40 \\
\hline $\begin{array}{c}\text { Per } \\
\text { Month/ } \\
\text { capita } \\
\end{array}$ & 22.726 & 5.907 & 0.351 & $\begin{array}{c}10434.1 \\
50\end{array}$ & 18.009 & 4.359 & 0.332 & 8172.00 & 14.770 & 2.135 & 0.253 & 6176.70 & 11.773 & 1.867 & 0.239 & 4996.35 & 3.350 & 0.282 & 0.290 & 1411.80 \\
\hline $\begin{array}{c}\text { Total per } \\
\text { day }\end{array}$ & 3.030 & 0.788 & 0.047 & 347.805 & 2.401 & 0.581 & 0.044 & 272.400 & 2.462 & 0.356 & 0.042 & 205.890 & 1.570 & 0.249 & 0.032 & 166.545 & 0.335 & 0.028 & 0.029 & 47.060 \\
\hline $\begin{array}{l}\text { Per day/ } \\
\text { capita }\end{array}$ & 0.758 & 0.197 & 0.012 & 3478.05 & 0.600 & 0.145 & 0.011 & 2724.00 & 0.492 & 0.071 & 0.008 & 2573.62 & 0.392 & 0.062 & 0.008 & 1665.45 & 0.112 & 0.009 & 0.010 & 352.950 \\
\hline Average & 90.905 & 23.628 & 1.403 & 55.605 & 72.038 & 17.435 & 1.328 & 47.263 & 73.848 & 10.675 & 1.265 & 26.710 & 47.093 & 7.468 & 0.955 & 17.132 & 10.050 & 0.845 & 0.870 & 6.540 \\
\hline Std. Dev. & 48.359 & 20.031 & 0.285 & 68.675 & 41.055 & 16.227 & 0.407 & 57.689 & 24.595 & 7.031 & 0.311 & 31.938 & 16.854 & 4.165 & 0.477 & 21.496 & 6.643 & 0.693 & 0.253 & 7.588 \\
\hline
\end{tabular}


Table 5:- Showing Total Average Solid Waste (Kg/Capita/Month) Generation and Composition in 5 Houses each from Zone I- Zone IV From June, 2007- May, 2008

\begin{tabular}{|c|c|c|c|c|c|c|c|c|c|c|c|c|c|}
\hline \multirow{2}{*}{$\begin{array}{c}\text { S.N } \\
\text { o. }\end{array}$} & \multirow{2}{*}{ Solid Waste } & \multicolumn{2}{|c|}{$\begin{array}{l}\text { Zone-I } \\
\text { H.1-H.5 }\end{array}$} & \multicolumn{2}{|c|}{$\begin{array}{l}\text { Zone-II } \\
\text { H.1-H.5 }\end{array}$} & \multicolumn{2}{|c|}{$\begin{array}{l}\text { Zone-III } \\
\text { H.1-H.5 }\end{array}$} & \multicolumn{2}{|c|}{$\begin{array}{l}\text { Zone-IV } \\
\text { H.1-H.5 }\end{array}$} & \multicolumn{2}{|c|}{$\begin{array}{c}\text { Total } \\
\text { H.1-H.5 }\end{array}$} & \multirow{2}{*}{$\begin{array}{c}\text { Total } \\
\text { A.V.*12 }\end{array}$} & \multirow{2}{*}{ \%age } \\
\hline & & A.v. & S.D. & A.v. & S.D. & A.v. & S.D. & A.v. & S.D. & A.V. & S.D. & & \\
\hline 1 & $\begin{array}{c}\text { Bio } \\
\text { Degradable } \\
\text { Solid Waste }\end{array}$ & 25.370 & 7.368 & 15.115 & 3.841 & 14.818 & 2.687 & 58.787 & 27.501 & 28.523 & 10.349 & 342.276 & 82.003 \\
\hline 2 & $\begin{array}{c}\text { Non- } \\
\text { Degradable } \\
\text { Solid Waste }\end{array}$ & 2.601 & 1.151 & 3.253 & 1.371 & 3.611 & 1.727 & 12.01 & 9.629 & 5.368 & 3.469 & 64.416 & 15.433 \\
\hline 3 & $\begin{array}{l}\text { Inert Solid } \\
\text { Waste }\end{array}$ & 0.764 & 0.347 & 0.671 & 0.152 & 0.967 & 0.363 & 1.164 & 0.346 & 0.892 & 0.302 & 10.704 & 2.564 \\
\hline 4 & $\begin{array}{l}\text { Total Solid } \\
\text { Waste }\end{array}$ & 28.735 & 8.866 & 19.039 & 5.364 & 19.396 & 4.777 & 71.961 & 37.476 & 34.783 & 14.120 & 417.396 & 100.00 \\
\hline
\end{tabular}

A.V. -Average

S.D.- Standard Deviation

3. Current waste disposal practices as followed by the municipality are unsatisfactory and may cause various environmental problems in the area of present investigations.

4. The improper solid waste disposal in open drains, vacant spaces and nallahs near the bank of river Chenab, may have an adverse impact on ground water as well as surface water quality.

5. In order to reduce the harmful impacts of solid waste on environment, proper scientific solid waste management practice is needed in the area so as to protect the living population from this menace of environmental concern.

\section{Acknowledgement}

The authors are highly thankful to the Department of Environmental Sciences, University of Jammu, Jammu for providing necessary facilities to undertake the present investigations.

\section{References}

Gupta, Subash C. and P. Manhas 2008. Percentage generation and estimated energy content of municipal solid waste at commercial area of Janipur, Jammu. Env. Conserv. J., 9 (1\&2): 27-31.

Gupta, Subash C. and S. Sambyal 2007. Solid Waste Management: A case study of Samba town of Jammu division of J\&K. Indian $\mathbf{J}$. Conserv. and Ecoplan, 14(3): 571-574.

Jaswal, S. 2008. Generation, composition, management and impact of Municipal Solid Waste on ground water quality at Muthi, Jammu. M.Phil. Dissertation submitted to the University of Jammu, Jammu.

Kour, J. 2004. Generation, disposal and management of solid waste in Jammu city. Ph. D. Thesis, University of Jammu, Jammu.

Krishan, K. 2010. Qualitative and quantitave analysis of commercial solid waste, estimated energy content and its management- A case study of Udhampur Town. M.Phil. Dissertation submitted to the Department of Environmental Sciences, University of Jammu, Jammu.

Rampal, R.K. and D. Sharma 2002. Generation and disposal of solid waste at Bagh-e-Bahu complex of Jammu. Indian J. Environ. And Ecoplan, 7(1): 51-54.

Rampal, R.K. and J. Kour 2005. Solid waste generation in the residential area of Jammu city (J\&k). Poll. Res.; 24 (2): 1-5. 


$$
8
$$

Environment Conservation Journal

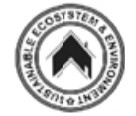

